\title{
The Mute, the Stoic and the Rebel: Animals in the Works of Mikhail Bulgakov and Nabarun Bhattacharya
}

\author{
Dibyakusum Ray
}

Assistant Professor in the National Institute of Technology, Silchar. ORCID: http://orcid.org/ooooooo2-9537-3277. Email: dibyakusum776@gmail.com.

Received May 30, 2016; Revised July 20, 2016; Accepted July 30, 2016; Published August 18, 2016

\begin{abstract}
This article attempts to trace the gradual 'otherification' of non-human entity, particularly animals, in Continental theory. This article would also explore how after presupposing the concept of subject as a human, with animals acting as "alive but no more" with no part in making judgments, Continental theory takes a turn. Levinas conceptualizes animals as "delightful" dociles facilitating human self-definition. Conversely, Derrida problematizes the multilayered man-animal/master-pet dialectics, as he points out the systematic exploitation of animals in society and artistic representation, as the animals are expected to be the mute receptacle of human vagaries - the perpetual 'other' who do not even speak or gaze back. Mikhail Bulgakov's The Master and Margarita and The Heart of a Dog, together with two of Nabarun Bhattacharya's works would serve as specific case studies to analyze the evolution of animal imagery from meek placebos through stoic indifference into a force of dissent-ever irreconcilable to the 'self.
\end{abstract}

Keywords: animal studies, aesthetics, ethics, Levinas, Derrida, Bulgakov, Nabarun Bhattacharya

...Can an animal be considered as the other that must be welcomed?...

...In the dog, what we like is perhaps his childlike character. ...even with regard to an animal, a pity. A dog is like a wolf that doesn't bite. ...Children are often loved for their animality. The child is not suspicious of anything. He jumps, he walks, he runs, he bites. It’s delightful. (Levinas, 1990, p.3)

\section{Introduction}

How does an animal see itself? This question is not meant to be posed as a test for the flexibility of human consciousness or perversely echoing Derrida's question of the autobiography of "The animal that I am," but in an effort to ascertain what position a non-human character is expected to have in a literary text. Is it perpetually relegated as the quintessential 'other', or is there any chance for a sentient animal to be acknowledged and included in a literary text? Or more precisely, is human consciousness equipped to sense and measure animal consciousness. And suppose, it does sense or acknowledge it, what is the power politics involved in this dialectics? Can an animal possibly be considered as a force of dissent in a human arena or does it disappear in a pitying assimilationst approach as cited by Levinas? This article will trace the gradual otherification of animals in literature through continental aesthetics, and the proliferation of non-

(c) AesthetixMS 2016. This Open Access article is published under a Creative Commons Attribution Non-Commercial 4.0 International License (http://creativecommons.org/licenses/by-nc/4.o/), which permits non-commercial re-use, distribution, and reproduction in any medium, provided the original work is properly cited. For citation use the DOI. For commercial re-use, please contact editor@rupkatha.com. 
human characters in the works of Mikhail Bulgakov, with certain cross reference to the works of Nabarun Bhattacharya, who Bulgakov have heavily influenced. In this discussion of the emergence of the 'other' as an irreconcilable force of dissent, a threefold argument on ontology will be explored - Kantian, Heideggerean and Levinasean.

Bulgakov has always been a major inspiration to Bhattacharya who claimed "manuscripts don't burn..." (Bulgakov, 2010, p. 326). It was famously quoted by the latter while dedicating Kangal Malshat to Bulgakov. Further, both writers addressed societies, with similar political ethos, concentrating on the mundane every day. Moreover, the use of animal figures from Bulgakov to Bhattacharya traverses a curious path playing with the master-pet dialectics, animals as docile placebos as well as manipulative mischievous imps in Bulgakov to the silent stoicism to irreconcilable resistance in Bhattacharya. Several of Bulgakov's seminal works consist of important animal characters, and their treatment of the issue of the self and other is probably the most distinct in and through such stories, especially as the animals being endowed a humanoid consciousness-- they speak, cry and feel while acutely conscious of their socio/political context and machinations of the human beings. Animals are symbols of the philosophical dynamics in these authors, as I will show through this essay, to be traced through a close analysis of three defining moments of continental theory: Emmanuelle Kant's subjective and universal good, Martin Heidegger's Dasein and the consumption of the other, Emmanuelle Levinas' divorce of the other from the self and subsequent responsibility, with special reference to Mikhail Bulgakov's "The Heart of a Dog", and a few short stories of the recently deceased underground writer Nabarun Bhattacharya.

\section{The Gradual Otherification of Non-human Entities}

Classical philosophy does not put animals just as an 'other' to the human but as a rhetorical invocation that appears to solve a problem: the problem of defining oneself (Derrida, 2002, p. 372). The article would only brush past Aristotle and Socrates, despite their frequent allusions to animal images. Aristotle denies animals the basic power of mimesis -- the faculty of observing and copying. In Plato's Phaedo, on the other hand, Socrates frequently compares himself with bee and gadflies, thereby descending to the level of bestiary. For them, animals are symptomatic analogies of life without consciousness, idiocy, demonic and the perpetual non-human, non-material 'other.' But to understand the gradual 'otherification', of the animals, we need to look at the construction of 'self as opposed to the idea of the 'other' in Continental Aesthetics.

Kant makes his argument in favour of universal perceptions of pleasure, judgment and goodness in, specifically, the first part of The Critique of Judgment. For Kant, nature (we can argue that it includes flora and fauna) is an apriori, represented to a subject through the representation of the object. The subject grasps the concept of an object through its sensuous faculties - a process Kant calls 'judgment,' and here starts the ubiquitous Kantian ambiguity (Kant, 1957, p. 9). Never solving, but merely explaining the paradox, Kant opines that our faculties are always in a dialectical position with representations - touch, sense, feel all are representative of our struggle of grasping what a concept is. It is this dialectics, this essential failure to conceptualize the concept which is generative of our emotions, that is universal (Kant, 1957, p. 11). To be very precise, Kant hardly considers any non-human entity as the subject passing the judgment. And human cognition towards any part of nature, including animals is universal and a part of a forcefully homogenous judgment. 
In Being and Time, Heidegger offers a concept of this absence of a concept - according to him, out of the Kantian dialectics only 'intentionality' makes the world meaningful to a subject. The subject is thus first of all an intrinsic part of the world, then it becomes conscious about it through its primary and unique concern with its own identity. The 'I' intends, and the world occurs. The apriori Dasein (the subject in relation with its milieu) is presupposed by the intentionality, yet he is conscious of his secondaryness (Heidegger, 1962, p. 12). Clearly, we are still struggling to maintain a very delicate balance between self and the other, the apriori nature and the aposteriori subject, in other words, the closure in a concept and the possibility of a dialogue. The moment the question of concern with one's own identity or the possibility of a dialogue comes in, we can assume that like Kant, Heidegger's subject is also human. The only time Heidegger mentions animals in Being and Time, is to distinguish between the Being and the being of an animal:

It remains a problem in itself to define ontologically the way in which the senses can be stimulated or touched in something that merely has life, and how and where the Being of animals, for instance, is constituted by some kind of "time." (Heidegger, 1962, p.396)

Animal for Heidegger is life but no more or nothing more (nur Lebenden). He refuses to accord any other sentience to animals than the virtue of being alive. Heidegger dubs animals as the "poor in the world", something he calls weltarm. They are without agency, without the power of any sentient faculty or even the power that is even allowed to the "other" -- they do not respond to the "self."

\subsection{Levinas' Ethics and "The Name of a Dog"}

Levinas' ethics, majorly in Totality and Infinity (1979) and Entre Nous (2007), arises from a further problematization of the subject, and herein we will also discover our cue into postcolonialism. "The idea of the infinite is found in my responsibility for the other (1979, p.113)," says Levinas, signalling that we are responsible beyond our intentions. Levinas opines that society and signification precede the impersonal structure of knowledge and reason, hence there is a state that is otherwise than being, or 'being's other.' The other is not auto-fathomable or autoconceptualized, and is in constant friction with the self. Levinas is never oblivious that a total replacement of the self by other would certainly result in the death of the consciousness, hence it exists in relation to the totality but remains within itself, separated from the totality. In his "The Name of a Dog" (1990) and the subsequent interview, Levinas puts human beings and animals as entities absolving each other of carelessness. For Levinas, human beings are the sentient beings who not only care for themselves, but can also care for the 'other', the certain responsibility towards the 'other' which defines the border of separation between the 'self and the 'other'.

Yet, for Levinas, the human face is the one which centrally posits the human face as the central point of the responsibility towards the 'other'. Human beings acknowledge the nonhuman other through a sense of pity or a delight in their servile, childlike non-ferality. Though Bobby the dog imparts the first acknowledgement to the systematically dehumanized Jewish inmates of the Nazi camp, by his "friendly growling” (p, 53), as human beings, Bobby's acknowledgement as the 'other' worthy to be recognized is, however, highly conditional. In the subsequent interview, Levinas opines that the dog's face, unlike the human face, is not pure. Despite his radical discussion about a non-human character, his Ethics, rests solely on human beings. For him, even animals have a hierarchy. The dog might have a face, but a snake does not. In "The Name of a Dog", the human face being capable of demands and supplications is an 
epiphany in itself. But the human face "deprived of everything because entitled to everything" is not the same face as the animal 'other.' The animal face, conversely, is not deprived of anything, because its entitlement is nil. It cannot have an epiphany in this context. Levinasean ethics do not acknowledge animal consciousness, thus. The best that the animal face can do is to recognize the human face as different from itself.

\subsection{Jacques Derrida: "What animal? The other ${ }^{\mathrm{ii}}$."}

Derrida (2002) ${ }^{\mathrm{iii}}$ starts it with quite a shock. He imagines himself naked and being frontally perused by a cat. His sense of 'shame' makes him question "Ashamed before what and naked before who?" (p.373) and concludes that he is ashamed being as naked as an animal. However, an animal cannot be naked just because it is. In order to be naked one needs to experience nakedness. The animal cannot be naked because it cannot sense or see itself being naked. Conversely, the human being's shame comes from his sentience of him'self and knowing himself to be ashamed ${ }^{\text {iv }}$. The animal will remain non-nude because it is un'self'consciously nude, and human beings will remain nude as it 'self consciously came out of it (p.374). Derrida leads us to the paradox of the situation, when we encounter the animal's eyes, do we get ashamed like the animal who has no sense of the nude, or the man who retains the sense of nudity? Who is the self then, and who does the self follow? Perversely, this can be answered only by the 'other', and in this case the cat.

Derrida alludes to Montaigne's "Ma Chatte" (My Cat), where Montaigne (2003) ridicules man's impudence in designating certain faculties and refusing certain other faculties to beasts. Montaigne argues that though man refuses imagination and presumption in beasts, the very power of some beasts to recognize letters and syllables entails certain consciousness and intelligence in the beast. It is curious to note that Montaigne's cat is categorically described as "My"/ his cat, though the ambivalence of the master-pet dialectics is also addressed". Further alluding to Alice in Wonderland and Through the Looking Glass, Derrida brings in the concept of a response from any communication with an animal. Alice is incredulous for not guessing an affirmative or a negative response from the cat, conversely admitting that she can guess such nature of response from a human subject. But when Derrida (2002) supposes himself being in any space where the cat can enter and see him naked, he supposes himself being alone with a cat, otherwise the source of his discomfort might be multiple. But he promptly asks whether anybody can be alone with a cat present? "In fact, is one ever alone with a cat? Or with anyone at all? Is this cat a third person? Or an other in a face-to-face duel?" (p.379). Human can see, observe, study or reflect on animals, but they, in turn, they are never, what Derrida says "seen seen" by the animal-their gaze never intersected. Theory so far has been like this:

...Descartes, Kant, Heidegger, Lacan and Levinas, belong to this quasi-epochal category. Their discourses are sound and profound, but everything goes on as if they themselves had never been looked at, and especially not naked, by an animal that addressed them... It is as if the men representing this configuration had seen without being seen, seen the animal without being seen by it, without being seen seen by it; without being seen seen naked by someone who, from the basis of a life called animal, and not only by means of the gaze, would have obliged them to recognize, at the moment of address, that this was their affair, their lookout... (Derrida, 2002, p.383)

The animal's position as the 'other' is more precarious that other categories of 'others', animals have no possibility of speaking back, nothing that will be registered or analysed. They are 
'wholly other.' In the Book of Genesis, man is ordered to command animals but not to name them. And when the turn for the public naming of animals comes, it is Adam or Ish, who does the honour, excluding Eve or Ishah. Even the animals that came into the world before them were named after them. And Ish would see and name them without allowing himself to be seen or named by them. This "infinite right of inspection" is accorded to the man. The animals are forever excluded from this right. They do not look back: "The animal is a word, it is an appellation that men have instituted, a name they have given themselves the right and the authority to give to another living creature [a l'autre vivantl]" (Derrida, 2002, p.392).

In the latter pages, Derrida offers his two hypotheses concerning the dialectics between human and animals. The first hypothesis concerns the violence toward animals over millenia as well as the ethical and political efforts to limit animal suffering. He alludes to the historical rituals of animal sacrifice, hectacombs ${ }^{\mathrm{vi}}$ and other exploitation of animal energy for human sustenance. In response to it, Derrida wishes to acknowledge and voice out the "philosophical problematic of the animal. ${ }^{\text {vii" }}$ Derrida refers to Jeremy Bentham's remarks on animal suffering, merging it with his own idea on radical passivity or curious vulnerability in the heart of power. More than the questions whether the animals can see or hear, it is should be asked whether they can suffer, thereby changing the axis of the logos or modality of enquiry. This war for the animals, according to Derrida, is a war of pity. The second hypothesis revolves around the "ontological" issues, questioning the criteria which determine human-animal distinction. He closes the essay with an analysis of the term "animot" instead of the heavily baggaged term "animal" to ensure the acknowledgement of the multiplicity of human-animal relationship. Derrida attempts to give some agency to the animals, beyond the concept of "animal figure" in fables and stories.

\section{Mikhail Bulgakov: The Master and Margarita and The Heart of a Dog}

Critics like Rita Pittman, Amy Nelson and Susan Ruth Mason opine that Bulgakov is obsessed with animals, dogs and cats in particular. In his works, animals are either the Heideggerian nonentities as a part of the landscape ${ }^{\text {viii; }}$ or they can be externalization of the soul of the human characters with whom they are associated ${ }^{\mathrm{ix}}$; or they can even be central characters in their own story. I would be focusing on the last.

Bulgakov's works, especially, The Master and Margarita and The Heart of a Dog have mostly been considered as allegories of contemporary Russian existence. In The Heart of a Dog the psychic confines of the book rests on the relationship between the dog and his master. Sharik's obedience to his master, Professor Preobrazhensky, is not left to contemplation: "I'll follow you wherever you like..." (Bulgakov, 2009, p. 12). Sharik, possibly, consciously gives up his autonomy as a stray accepting domestication. He accepts the human rule of dominion over the non-human subjects submissively, as well as naturalizes the domination. Further, his getting collared by his master imparts a sense of going up in the world-a rise in status that allows him access in places hitherto denied to him. His domestication by a human and its representation through the collar is glorifying for this animal, as it internalises the dominion that a totalitarian society might subject a human to. However, the master-pet dialectics (with all its patronizing, hierarchical and maternal/paternal strains) is hampered as the pet is experimented on without its consent and literally turned into a human-Sharikov. In a Frankensteinian attempt in playing God, the master does the unthinkable to improve mankind. He transplants the pituitary gland and testicles of a deceased convict to turn Sharik into a man. It is curious that Sharik's growth and masculinity is preserved without any question, but his inherent bestiality is not addressed at all, 
as if it is of no consequence. The human race, here has turned into an impotent, phallogocentric, monstrous construct engaged in using animals as objects, though they recognize their significance as sentient beings. Animals, in The Heart of the Dog, are the ultimate subaltern who speak, but are ritualistically silenced.

In The Master and Margarita, however, Bulgakov uses animals in all the three ways. They are the processions of mute animals with nothing to add to the scenario other than being the Heideggerian "alive but no more"; or they are the myriad of animals that margarita comes across on her way back from the Satan's Ball; or they are like the giant cat Behemoth who acts as one of the central characters in the novel. Behemoth is almost the Shakesperian Fool, who plays the animal but is the wisest and the most observant of the follies of mankind. Pilate's dog Banga, Behemoth and the police dog Tuz Buben are not mute. Their personalities are clear as the noble Banga corresponds to the mischievous Behemoth and the simple dog Tuz Buben. Banga is the true friend to mankind like Sharik the dog, while Behemoth is the crafty misanthrope, conscious of his status in human awareness:

Actually, I do happen to resemble a hallucination. Kindly note my silhouette in the moonlight." The cat climbed into the shaft of moonlight and wanted to keep talking but was asked to be quiet. "Very well, I shall be silent," he replied, "I shall be a silent hallucination. (Bulgakov, 2010, p.376)

Bulgakov's Behemoth is magical and is aware of it. The mankind that he associates with expects him to be mute, and he plays along as he manipulates them effectively.

\section{Nabarun Bhattacharya: "Andha Beral” and Lubdhak}

"Andha Beral" or "The Blind Cat" [my translation] was published in 1997 in the magazine Pratikshan. The whole story comes to the reader as the purely instinctive impression of a world moving along with its own dynamics, centring the sole figure of non-human entity that has nowhere to go, nothing to do, nothing to aspire for. Surviving is the only pure drive for the blind cat that sits almost unmoving under a derelict table in a derelict small own beside a river. No other concrete information of the setting is ever provided in the story, all we know that:

... the chances of anybody remembering the blind cat are extremely few. ...Many, many people-all of them so busy with themselves that they never realized towards the slope of the floor in some gap amongst the crooked tables and chairs the blind cat is sitting, ears upright. He couldn't understand all the words, the events, the sounds of shattering glass, that sound of the burning cigarette stubs touching the river's surface, songs, belching, shards of laughter, halting sobs. There is a difference between hearing and understanding. [my translation] (Bhattacharya, 2006, p. 185)

Not only the does blind cat sit there unstirring and unmeddling, but he also can neither see nor fully grasp every significance of all that goes on surrounding him; thus making him a witness who shall never turn into an actor. He is the symptomatic mute for Aristotle and Socrates, the alive and no more for Kant and Heidegger. He does not even acknowledge the humanity of the human beings like Levinas' Bobby. The machinations or purposefulness of the blind cat's mind is never revealed.

Another important aspect is the interplay of myriad of emotions triggered by the presence of the cat-sympathy, nonchalance, heartbreak or simply innocent and evasive curiosity, all 
encircle fruitlessly the pure survival instinct of the blind cat-nothing makes any difference the way he spends each moment. The personal anguish or the tragedy of a revolutionary cease to matter as the universe is actually like the blind cat-constantly anticipating, constantly listening but never coming to a conclusion that can be called satisfactory. Meanwhile, the 'story' meanders into the petty small talks among the government officials conducting the workshop in the village, and the detail that is revealed out of the discussion possibly serves as the only back story of this particular narrative:

There was an experiment involving seven newborn kittens in England. It was conducted by the defense ministry... The blind cat did hear this, but couldn't comprehend... but assault can come in any dark recess. Maybe not like the seven unfortunate kittens, but it can never be predicted what dangerous fate awaits the blind cat. And, it might not necessarily come from the humans as well. [my translation] (Bhattacharya, 2006, p. 188)

Bhattacharya's use of animal imagery breaks the boundary of subjectivity, as the blind cat is neither one nor the other, it is just the Sentient 'is'. It registers motion but refuses to react. This prepares us for the physical ending of the story. The author all of a sudden shifts into future tense and reveals that there will be a tremendous thunder storm in the next few days. It is predicted that if the water breaks into the hotel, the best the blind cat can do is to climb up a little towards the opposite side of the room but it will certainly not go out. The fate of the blind cat is not important. "When the kids will come in the morning, they will discover him sitting silently in the familiar place [my translation] (Bhattacharya, 2006, p.189)". The author employs this uncertainty of personal fate as an essential device to grasp a fleeting impression of the nameless, faceless entity that sits in the centre: it is not a sadness of or for anything specific, rather, this melancholia only guides us to the realization that the icy vastness of the network of beings is something which is not necessarily concerned with personal tragedies.

Lubdhak or Sirius [my translation] (2006) incorporates another, possibly final, dimension to Bhattacharya's philosophy of life - intentionality, responsibility and a consciousness that, in its rejection of status quo, might hint of a new beginning. Sirius talks about a dystopic Calcutta in its throws of overpopulation, oppression and grim futuristic urbanity. Ignorant of the fact of its imminent destruction by a rogue asteroid projectiled from the constellation Canis Minor, the Calcuttans immerse themselves into a radical activity of beautifying the city-liquidating the ghettoized urban population. The stray dog community of Calcutta becomes the first casualty. Dogs, already tortured, mutilated, blinded or maimed by a million unkind instances, are concentrated into kennels of the British era-left to die without food or other means. Although resilient at first, the dogs suddenly allow themselves to be caught, and take part in mysterious dance like movements inside the kennels under the watchful eye of close-circuit cameras. As the bafflements of the human scientists increase, the dog-ritual intensifies though many perish of exhaustion, and it is suddenly understood that they in their classless, divisionless mass consciousness, have realized the imminent destruction of the city and the possibility of a new beginning. Finally although the humans remain in darkness, the old dogs let the young free, streets of Calcutta are flooded with migrating puppies that abandon the dystopic city in search of what... the author never explains. The use of animals is not symbolic of the Sentient anymore. The animals react. They participate and protest in the face of cosmic and human annihilation. They are no longer docile non-entities, figures of pity or symbols of something greater. The dogs talk, emote, swear and take oaths:

The huge buildings, feet covered in merciless boots, the wheels of a raging car ... these, that instil layers of fear inside us, that make us understand that its us who are the 
weaklings-all of these are now silent, cornered and still. Woof! Woof! We bark in small voices. Yes, today we control it all... we are leaving ... We leave in stride. You may guard your assets in this cursed city we rejected, like blind Golems... Your cruelty, ignorance, greed, callousness are all coming back to you like boomerangs... if the police sergeant moves one bit, his feet will hit us and he will never ever reach home tonight. All the trucks ... beware! Your wheels must not move an inch, for this placid, vast, sea of canines might turn into a gaping shark... Woof! Woof! [my translation] (Bhattacharya, 2010, pp. 411-412) ${ }^{\mathrm{xi}}$

\section{Conclusion}

The challenge is to tether the seemingly disparate strands of this argument-the evolution of animals from the mute to the irreconcilable 'other'. We start from a merger of several dimensions all primed to fight for a singular cause-which generally ends in animal characters described as sympathetic bystanders as in Heidegger or docile placebos as in Levinas. Their existence or acknowledgement of it is still contingent to human perception or empathy. Their self-hood is never established. In the second phase, we have a much more defined and multi-faceted animal existence that, finally, reveals itself to be the sightless, inert singularity that presupposes and realizes the machinations of the universe. The animal (Bulgakov's Sharik/ Sharikov) acts as the perfect foil for the monstrous and impotent human race. They are a symbol for the Sentient "is"registering all the movements but choosing not to react to anything like Bhattacharya's blind cat. The third phase, however, endows the animals the status of the layered, conflicted 'other' that rejects the self in favour of a space of their own, as in Derrida. Misanthropic Machiavellis like Behemoth, whose muteness is a ploy for manipulating mankind, and the rebellious dogs in Lubdhak unabashedly place themselves as the 'other' who will never reconcile with the hegemonic power structure. The real ontological violence against the 'other' comes from the attempt to equate it as part of the 'self - the 'other' identity is not to be shunned or mutated into an organic whole; it is the ability to say 'no' to the normative impulses is what makes Bulgakov and Bhattacharya's animal characters that travel from the first to the third phase, the most remarkable of them all.

\section{Notes}

${ }^{\mathrm{i}}$ We cannot but cross refer with books like Issac Asimov's I Robot and Bicentennial Man, where the sentience of robots is questioned. Albeit, their thoughtful rendition of non-human characters, the robots are acknowledged as sentient, altruistic beings as they are gradually humanized. Being a human is the yardstick which every non-human should aspire to.

ii Derrida, 2002, p. 373.

iii Derrida first delivered the written text of "The Animal That Therefore I Am" at a conference in Cerisy, France in 1997.

${ }^{\text {iv }}$ Derrida posits modesty as a human attribute. Human beings are conscious of their 'selves', and hence can feel shame, but animals cannot experience this shame. Does that take the sense of 'self from the animal consciousness:

We would therefore have to think shame and technicity together, as the same "subject." ...Man would be the only one to have invented a garment to cover his sex. He would only be a man to the 
extent that he was able to be naked, that is to say to be ashamed, to know himself to be ashamed because he is no longer naked. And knowing himself would mean knowing himself to be ashamed. On the other hand, because the animal is naked without consciousness of being naked, modesty would remain as foreign to it as would immodesty. As would the knowledge of self that is involved in that. (Derrida, 2002, p.374)

$\checkmark$ "When I play with my cat, who knows if I am not a pastime to her more than she is to me?" (Montaigne, 2003, p. 401)

vi The ritual of slaughtering one hundred cattle of any specie.

vii "In response to the irresistible but unacknowledged unleashing and the organized disavowal of this torture, ...in order to awaken us to our responsibilities and our obligations with respect to the living in general, and precisely to this fundamental compassion that, were we to take it seriously, would have to change even the very basis ...of the philosophical problematic of the animal." (Derrida, 2002, p. 395)

viii In The Master and Margarita there are instances of the camels and donkeys trotting outside Pilate's house in ancient Jerusalem to signify an impending apocalypse. They do nothing but add to the realistic depiction of the scene. Likewise in his story "The Blizzard", Bulgakov uses a steady entry of wolves and horses, to add to the scene.

ix The practice of using animals and animal imagery to comment on the human characters, is a rather over used practice. Right from Plato through Philip Pullman to Julia Lee, writers and artists have made use of animal imagery to externalize the inner psyche of the human characters with their cheer, frustration, angst, optimism or desolation. Bulgakov uses the image of a mouse nibbling at a rind of cheese to depict the inherent meanness of Vasilisa's wife in The White Guard. On the other hand, in A Theatrical Romance, the image of the cat is a much used trope. The frightened cat can stand for a nervous actress, can represent the writer's loneliness; and can accentuate his depression with its untimely death.

x ....েেই হিলেবে বলা যায় অন্ধ বেড়ালকে কতজনই-বা চেনে যে মনে রাখবে? ওই হোটেলে কত কত লোক খেয়েছে, থেকেছে, খুন করেছে, আত্মহত্যা করেছে, বোকা নেয়েদের বিয়ে করব বলে নিয়ে এসে মস্তি করেছে, মাছের ব্যবসার হিসেব কষেছে ...এরকম কত, কত লোক - এরা নিজেদের নিয়ে এতই ব্যাস্ত ছিল যে খেয়ালই করেনি মেঝের নিচু দিকটায়, জোড়াতালি টেবিল চেয়ারগুলোর এক ফাঁকে অন্ধ বেড়াল কান খাড়া করে বসে রয়েছে। এমন নয় যে কথাগুলো বা ঘটনা বা কাচের শব্দ বা দোতলার জানলার ফোঁকর দিয়ে বাইরে, জলের দিকে ছুঁড়ে দেওয়া সিগারেটের শেষটা জলে ছোয়াঁনাত্র ছ্যাঁক করে যে শব্দ হয় বা কোনো গান, কোনো টুকরো কথা, টেঁকুর, খুচরো হাসি, গুঙিয়ে গুঙিয়ে কান্না-এর সবগুলোর মানে সে বুঝতে পেরেছিল। শোনা আর বোঝার মধ্যে ফারাক থাকে। (শ্রেষ্ঠ গল্প, ১৮-৪-

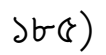

xi ... বড় বড় বাড়ি, বুটজুতো-পরা পা, গাড়ির রাক্ষুসে চাকা... এই সবকিছুই যার মধ্যে পরতে পরতে ভয় মিশে আছে, যা সবসময় বুঝিয়ে দেয় যে তোরা ফালতু, দুবলা, ভীতু... সেই ভয় দেখানো সমকিছু কেনন চুপ, কোণ্ঠাসা ও অচল হয়ে পড়েছে। কাউ! কাউ! এই তো আমরা ছোট ছোট গলাতেই ডাকছি। হ্যাঁ, সবকিছু আজ আমাদের দখলে... আমরাই চলে যাচ্ছি। তবে মাথা নিচু করে নয়। সসম্মানে। তোমরা বলে বসে আমাদের প্রত্যাখ্যানে অভিশপ্ত শহরে অন্ধ যখের মতো এবার তোমাদের ধন-সম্পদ আগলাও... তোমাদের নিষ্ঠুরতা, তোমাদের অবজ্ঞা, তোমাদের নির্মমতা, তোমাদের লোভ, তোমাদের অজ্ঞতা বুনের্যাং হয়ে ফিরে আসছে... পুলিশ সার্জেন্ট, তুনি খিলান বা দরজার মতোই দাঁড়িয়ে থাক। নড়লেই আমাদের গায়ে পা লাগবে। এবং ঘটনাটা যদি একবার ঘটে তাহলে আজ কেন, আর তোনার কখনই বাড়ি ফেরা হবে না। ...যত লরি আটকে গেছে তার চালকেরা সাবধান। একটা চাকাও যদি একটুও গড়ায় তাহলে এই বিশাল শান্ত সমুদ্র কিন্ত একটা বিরাট হাঙর হয়ে হাঁ করবে...কাউ! কাউ! (উপন্যাস সমগ্র, ৪১১৪১২) 


\section{References}

Acampora, Christa Davis and Ralph R. Acampora (eds.). (2004). A Nietzschean bestiary: Animality beyond docile and brutal. Lanham, MD: Rowman and Littlefield.

Armstrong, Susan J. and Richard G. Botzler (eds.). (2003). The animal ethics reader. New York: Routledge.

Beauchamp, Tom L. and R. G. Frey (eds.). (2011). The oxford handbook of animal ethics. New York: Oxford University Press.

Bhattacharya, Nabarun. (2006). Sreshtho galpa. Kolkata: Dey's Publishing.

---. (2010). Upannyas samagra. Kolkata: Dey's Publishing.

Buchanan, Brett. (2008). Onto-ethologies: The animal environments of Uexkuell, Heidegger, Merleau-Ponty, and Deleuze. Albany, NY: SUNY Press.

Bulgakov, Mikhail. (2009). The heart of a dog, trans. Michael Glenny. London: Vintage Books.

---. (2010). The master and Margarita, trans. Michael Glenny. London: Vintage Books.

Calarco, Matthew. (2008). Zoographies: The question of the animal from Heidegger to Derrida. New York: Columbia University Press.

---. and Peter Atterton (eds.). (2004). Animal philosophy: Essential readings in continental thought. London: Continuum.

Derrida, Jacques. (2002). "The animal that therefore I am (More to follow)," trans. David Wills. Critical Inquiry, 28(2), pp. 369-418.

Heidegger, Martin. (1962). Being and time. London: Wiley-Blackwell.

Krell, David Farrell. (2013). Derrida and our animal others: Derrida's final seminar, the beast and the sovereign. Bloomington: Indiana University Press.

Levinas, Emmanuel. (1990). "The name of a dog, or natural rights." Difficult freedom, trans. Sean Hand. Baltimore: John Hopkins University Press.

Montaigne, Michel de. (2003). The complete works, trans. Donald M. Frame. London: Everyman.

Sandoe, Peter, Sandra Corr and Clare Palmer. (2016). Companion animal ethics. West Sussex: John Wiley \& Sons.

Steeves, H. Peter (ed.). (1999). Animal others: On ethics, ontology, and animal life. Albany, NY: State University of New York Press. 